\title{
Influence of jets and resonance decays on the constituent quark scaling of elliptic flow
}

\author{
E E Zabrodin $\dagger \ddagger$, L V Bravina $\dagger$, G Kh Eyyubova $\dagger$ †, \\ I P Lokhtin $\ddagger$, L V Malinina $\ddagger \S$, S V Petrushanko $\ddagger$ \\ A M Snigirevł \\ $\dagger$ Department of Physics, University of Oslo, Oslo, Norway \\ $\ddagger$ Skobeltsyn Institute of Nuclear Physics, Moscow State University, Moscow, \\ Russia \\ $\S$ Joint Institute for Nuclear Researches, Dubna, Moscow Region, Russia
}

\begin{abstract}
The Monte Carlo HYDJET ++ model, that contains both hydrodynamic state and jets, is applied to study the influence of the interplay between soft and hard processes on the formation of the elliptic flow in heavyion collisions at RHIC and LHC energies. Jets are found to cease the hydro-like increase of the elliptic flow with rising $p_{T}$. Since jets are more influential at LHC than at RHIC, the $v_{2}$ at LHC should be weaker than that at RHIC. Violation of the number-of-constituent-quark (NCQ) scaling is predicted. The decays of resonances are found to enhance the low- $p_{T}$ part of the elliptic flow of pions and light baryons, and work toward the fulfillment of the NCQ scaling. PACS numbers: 25.75.-q, 25.75.Ld, 24.10.Nz, 25.75.Dw
\end{abstract}

\section{Introduction}

The elliptic flow of hadrons, produced in the course of non-central heavy-ion collisions, is considered as one of the main probes of the reaction early stage. It originates from the rescattering process in the initially anisotropic overlapping region. Recall that elliptic flow is the second coefficient of Fourier decomposition of particle invariant cross section in the azimuthal plane, namely $v_{2}=\langle\cos (2 \phi)\rangle \equiv\left\langle\left(p_{x}^{2}-p_{y}^{2}\right) / p_{T}^{2}\right\rangle$. Here $\phi, p_{T}, p_{x}$, and $p_{y}$ are the azimuthal angle, particle transverse momentum, and its in-plane and out-of-plane components, respectively. The spatial anisotropy of the almond-shaped area is then transformed to the anisotropy in the momentum space. When the isotropy in the coordinate space is restored, the elliptic flow stops to develop anymore. Thus $v_{2}$ is linked to the equation of state (EOS) of hot and dense partonic matter formed in the very beginning of the collision.

Many features of the flow at energies up to top RHIC energy $\sqrt{s}=200 \mathrm{AGeV}$ are successively reproduced by several models, in particular, hydrodynamic models (for review, see [1] and references therein). However, predictions of these models for the elliptic flow at top LHC energy $\sqrt{s}=5.5 \mathrm{ATeV}$ often contradict each other. For instance, simple scaling model 2 favors increase of $v_{2}$ at midrapidity to $v_{2}(y=0) \approx 7-8 \%$, several models predict saturation of the flow [2, whereas ideal hydro indicates [3,4] that $p_{T}$-integrated flow at LHC will be larger than that at RHIC although $v_{2}^{\mathrm{LHC}}\left(p_{T}\right) \leq v_{2}^{\mathrm{RHIC}}\left(p_{T}\right)$ in the domain of low and intermediate transverse momenta. 
Jets contribute to the elliptic flow via the so-called jet quenching mechanism that accounts for the quite weak flow of about $5 \%$. The simultaneous description of the flow caused by the soft processes and the jets, and study of the interplay between these two mechanisms has been done recently in [5] by means of the HYDJET++ model [6. The model contains the soft physics part, represented by the parametrized hydrodynamics with given freeze-out conditions [7], and the jets. The production of hard multiparton states and their propagation in dense partonic matter takes into account radiative and collisional losses [8] associated with the parton rescattering in the expanding QGP. The contribution of both soft and hard parts to the total multiplicity of a heavy-ion collision at ultrarelativistic energies depends merely on the collision energy and centrality. The few free parameters of the model have been tuned to provide adequate description of hadron spectra, elliptic flow, femtoscopic momentum correlations, and high $p_{T}$ tails of hadron spectra in gold-gold collisions at RHIC energies. Further description of the HYDJET ++ model can be found in [6] 8 . The main aim of our present paper is the study of the interplay between soft and hard processes responsible for the elliptic flow production in heavy-ion collisions at energies of RHIC and LHC, respectively. The influence of the resonance decays on the final elliptic flow of particles is very important at both energies in question. Here, we are going to benefit from the rich table of baryon and meson resonances (about 360 states) implemented in the HYDJET++ model.

\section{The influence of jets and resonances on the $v_{2}$ formation}

The model description of the particle elliptic flow as a function of the transverse momentum is plotted in Fig. 1 onto the RHIC experimental data taken at $\sqrt{s}=$ $200 \mathrm{AGeV}$. Besides the standard rise of the flow excitation functions and the mass ordering of the particle flow at $0 \leq p_{T} \leq 2 \mathrm{GeV} / c$, the model exhibits also the

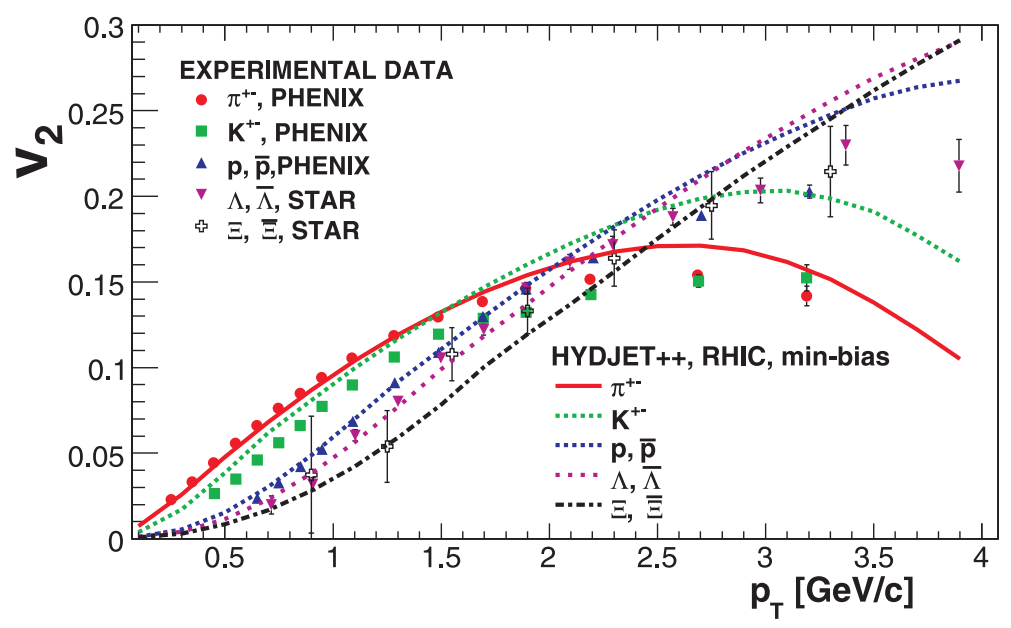

Figure 1. The $v_{2}\left(p_{T}\right)$ dependence in the HYDJET ++ model for different hadron species (lines) and comparison with RHIC data (symbols).

saturation and the drop of the particle $v_{2}\left(p_{T}\right)$ at $p_{T} \geq 2.5 \mathrm{GeV} / c$ accompanied by the violation of the mass ordering. The last two features cannot be attributed to ideal hydrodynamics that indicates the instant rise of the elliptic flow up to unity 
without the crossing of meson and baryon branches as $p_{T}$ rises to infinity. Here we see the result of the interplay between the soft hydro processes and hard jets. In contrast to hard processes, the multiplicity of hadrons produced in soft processes drops exponentially with increasing transverse momentum. Therefore, after a certain $p_{T}$ the particle spectrum is dominated by the jet particles. The latter carry quite weak elliptic flow, thus the combined flow of high $p_{T}$ hadrons drops. Also, the slope of the $p_{T}$ spectra of heavy hadrons is not as steep as that of the light particles. Because of this, the hydro component of the transverse momentum distribution of heavy particles dominates until larger values of $p_{T}$ resulting to the change of the mass ordering of the flow: After a certain $p_{T}$ the heaviest particles possess the largest flow.

What are the consequences of such peculiarity in the behavior of elliptic flow for the heavy-ion collisions at LHC energies? Will the hadronic elliptic flow extend its strength or not? To answer these questions we generated 1,000,000 $\mathrm{Au}+\mathrm{Au}$ collisions at $\sqrt{s}=200 \mathrm{AGeV}$ and $500,000 \mathrm{~Pb}+\mathrm{Pb}$ collisions at $\sqrt{s}=5.5 \mathrm{ATeV}$, both reactions at fixed centrality $c=42 \%$. The centrality was chosen to observe already a strong elliptic flow and, simultaneously, to keep the flow fluctuations at a rather modest level. Transverse momentum dependence of elliptic flow of charged pions, (anti)protons, charged kaons, and $\Lambda+\Sigma$ is displayed in Fig. 2 for $\mathrm{Au}+\mathrm{Au}$ and $\mathrm{Pb}+$ $\mathrm{Pb}$ collisions at RHIC (left panels) and LHC (right panels) energies, respectively. One
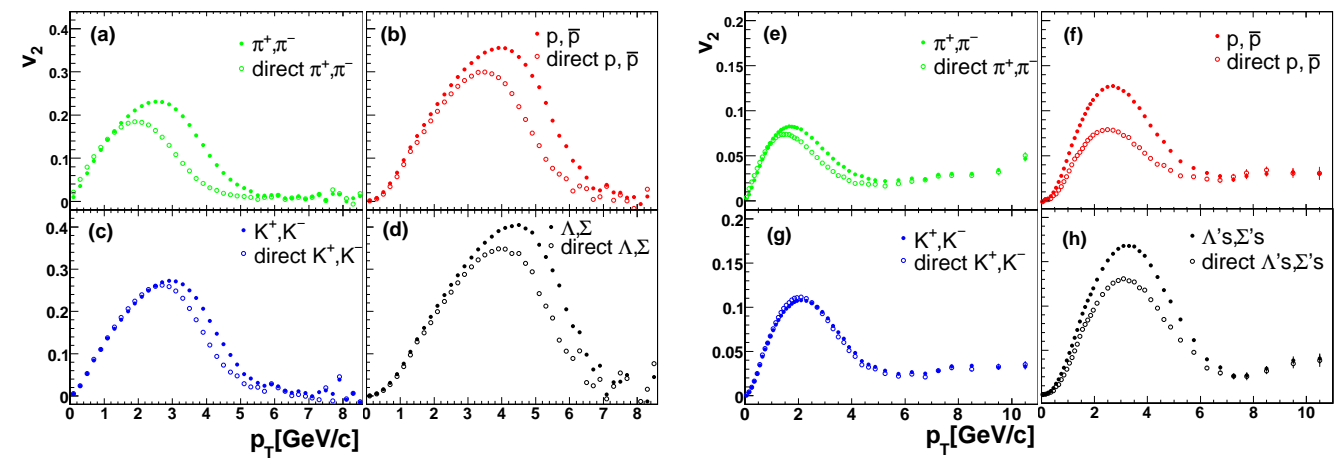

Figure 2. Left: The $v_{2}\left(p_{T}\right)$ dependences of all hadrons (full symbols) and of direct hadrons (open symbols) in the HYDJET ++ simulations of $\mathrm{Au}+\mathrm{Au}$ at $\sqrt{s}=200 \mathrm{AGeV}$ with centrality $c=42 \%$ : (a) $\pi^{c h}$, (b) $p+\bar{p}$, (c) $K^{c h}$, and (d) $\Lambda+\Sigma$. Right: Similar distributions, but for $\mathrm{Pb}+\mathrm{Pb}$ collisions at $\sqrt{s}=5.5 \mathrm{ATeV}$; $(\mathrm{e}),(\mathrm{f}),(\mathrm{g}),(\mathrm{h})$ correspond to $(\mathrm{a}),(\mathrm{b}),(\mathrm{c})$ and $(\mathrm{d})$, respectively.

can see that the flow of the most abundant hadron species at RHIC is stronger than its LHC counterpart. Although the soft hydro parts of the elliptic flow at RHIC and at LHC are similar [5], the hard parts are not. More particles are produced at LHC via the jet fragmentation. Therefore, hadrons from jets start to dominate over the hadrons, originated from the soft processes, at lower $p_{T}$ as compared with RHIC. The resulting elliptic flow at LHC is thus severely reduced. Another fact distinctly seen in Fig. 2] is the influence of the resonance decays on the elliptic flow of directly produced particles. Note that according to the model calculations, resonances contribute to the production of $80 \%$ of pions, $70 \%$ of protons, $60 \%$ of $\Lambda+\Sigma$, and more than $50 \%$ of kaons at LHC. For all hadrons, except kaons at LHC, final state interactions (FSI) increase the particle flow in the interval $1.5 \mathrm{GeV} / c \leq p_{T} \leq 6 \mathrm{GeV} / c$. However, decays of resonances diminish the pion flow at $p_{T} \geq 1.5 \mathrm{GeV} / c$ for both energies in question. 
The contributions of $\Delta$ decays to the flows of pions and protons, and $\rho$ and $\omega$ decays to pion elliptic flow are presented in Fig. 3. Elliptic flows of all resonances are weaker than that of pions at low transverse momenta and stronger at $p_{T} \geq 1.5 \mathrm{GeV} / c$.
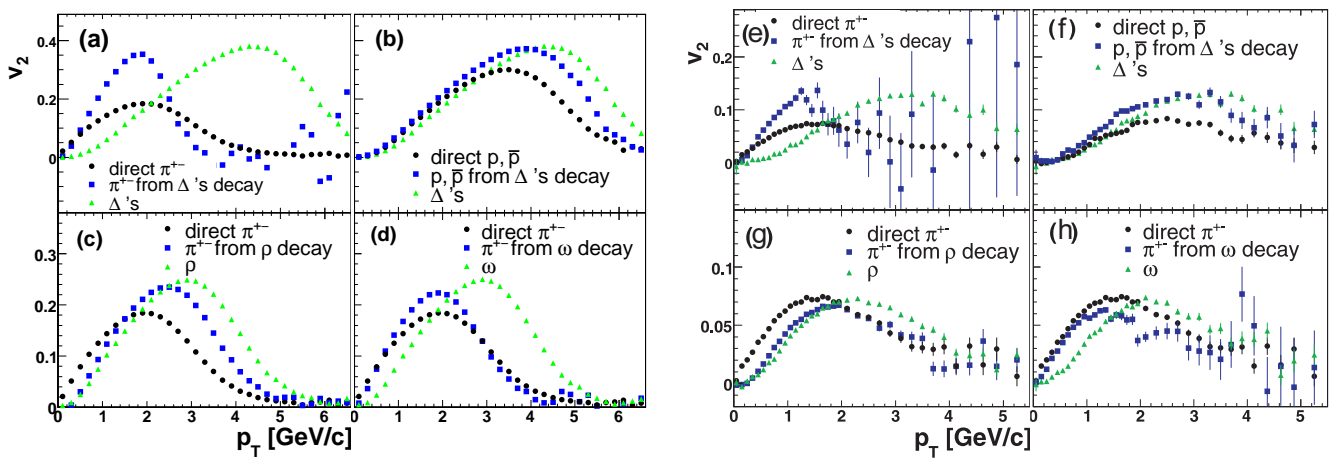

Figure 3. Left: The $v_{2}\left(p_{T}\right)$ dependence for (a) $\pi^{c h}$ and (b) $p+\bar{p}$ produced both directly (circles) and in $\Delta$ decays (squares) in the HYDJET ++ simulations of $\mathrm{Au}+\mathrm{Au}$ at $\sqrt{s}=200 \mathrm{AGeV}$ with centrality $c=42 \%$. (c) and (d) The same as (a) but for charged pions produced in decays of $\rho$ and $\omega$, respectively. The flow of resonances is shown by triangles. Right: Similar distributions, but for $\mathrm{Pb}+$ $\mathrm{Pb}$ collisions at $\sqrt{s}=5.5 \mathrm{ATeV} ;(\mathrm{e}),(\mathrm{f}),(\mathrm{g}),(\mathrm{h})$ correspond to (a),(b),(c) and (d), respectively.

Due to the decay kinematics, pions from the decay $\omega \rightarrow 3 \pi$ are getting softer $p_{T}$ distributions compared with that of $\omega$. In contrast, the transverse momentum distributions of pions from the $\rho \rightarrow 2 \pi$ decay are close to that of the $\rho$ meson. As a result, elliptic flow of pions from the $\omega$ decay is transferred to the softer $p_{T}$ region compared with $v_{2}^{\omega}\left(p_{T}\right)$, whereas elliptic flow of pions from the $\rho$ decay is similar to $v_{2}^{\rho}\left(p_{T}\right)$. Decays of $\Delta$ 's produce protons with transverse momenta close to that of the mother particles and pions with rather soft $p_{T}$ spectra. Again, the elliptic flow of pions is shifted to softer $p_{T}$ region. Therefore, the resulting elliptic flow of pions is a bit lower than the $v_{2}$ of directly produced pions at $p_{T} \geq 1.5 \mathrm{GeV} / c$ and stronger at intermediate transverse momenta. Modification of the elliptic flow of pions and light baryons can lead to violation of the hydro-induced mass hierarchy in the $v_{2}\left(p_{T}\right)$ sector. Particularly, the so-called number-of-constituent-quark (NCQ) scaling [9, 10 can be violated. Below we check this hypothesis.

\section{Violation of NCQ scaling}

The NCQ scaling claims the similarity of the $v_{2} / n_{q}\left(K E_{T} / n_{q}\right)$ distributions, where both the elliptic flow $v_{2}$ and the transverse kinetic energy of a hadron $K E_{T}$ are divided by the number of constituent quarks $n_{q}$. This scaling holds up until $K E_{T} / n_{q} \approx 1 \mathrm{GeV}$ 11. The HYDJET ++ calculations of the $v_{2} / n_{q}\left(K E_{T} / n_{q}\right)$ in heavy-ion collisions at RHIC and LHC are shown in Fig. 4 both for directly produced particles and for all hadrons. One can see that for direct hadrons the NCQ scaling is approximately satisfied at $K E_{T} / n_{q} \leq 1 \mathrm{GeV}$ at RHIC only. At LHC the NCQ scaling is not achieved for the direct particles due to the strong influence of the jets. To see possible deviations more distinctly, the particle distributions are also normalized in Fig. 4 to the elliptic flow of lambdas, $v_{2}^{h} / n_{q}^{h}: v_{2}^{\Lambda} / 3$. 
Influence of jets and resonance decays on the constituent quark scaling of elliptic flow 5
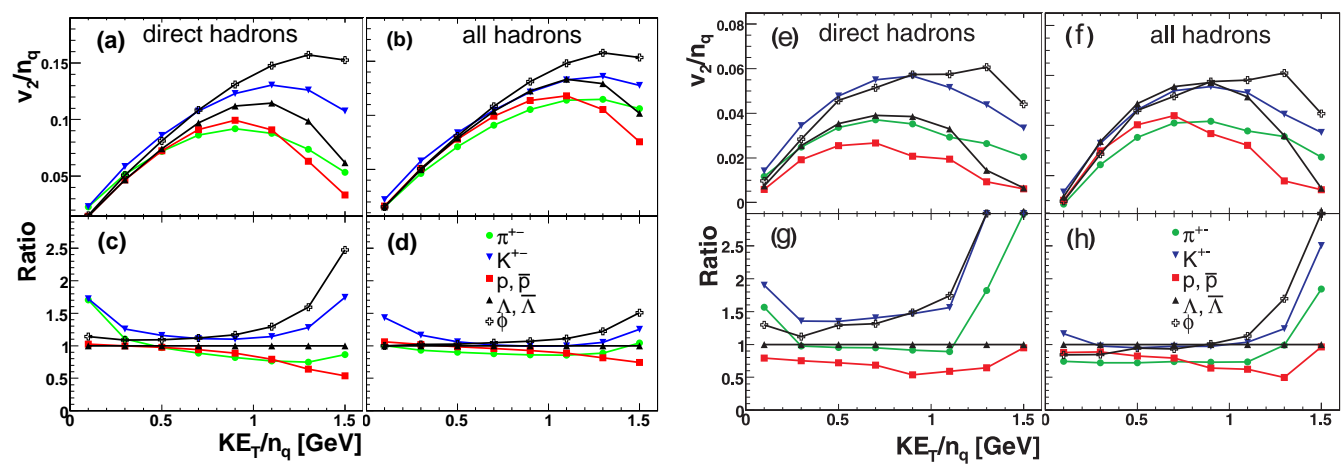

Figure 4. Left: Upper row: The $K E_{T} / n_{q}$ dependence of elliptic flow for (a) direct hadrons and (b) all hadrons produced in the HYDJET ++ simulations of $\mathrm{Au}+\mathrm{Au}$ at $\sqrt{s}=200 \mathrm{AGeV}$ with centrality $c=42 \%$. Bottom row: The $K E_{T} / n_{q}$ dependence of the ratios $\left(v_{2} / n_{q}\right) /\left(v_{2}^{\Lambda} / 3\right)$ for (c) direct hadrons and (d) all hadrons. Right: Similar distributions but for $\mathrm{Pb}+\mathrm{Pb}$ collisions at $\sqrt{s}=5.5 \mathrm{ATeV} ;(\mathrm{e}),(\mathrm{f}),(\mathrm{g}),(\mathrm{h})$ correspond to (a),(b),(c) and (d), respectively.

The decays of resonances essentially improve the situation. For instance, the elliptic flow of $\phi$ mesons has no resonance feed-down, whereas the flows of protons and lambdas are significantly enhanced by heavy resonances at $K E_{T} \geq 0.5 \mathrm{GeV}$. Because of the resonance decays, the NCQ scaling holds at $10 \%$ up to $K E_{T} \geq 1.0 \mathrm{GeV}$ for RHIC energies. At LHC the effect of jets is too strong, and only the approximate number-of-constituent-quark scaling is realized.

\section{Conclusions}

In conclusion, the interplay between hydrodynamic processes and jets, and its influence on the formation of the particle elliptic flow is studied within the HYDJET ++ model for heavy-ion collisions at RHIC and LHC energies. Jet particles that carry quite weak elliptic flow start to dominate over the hydrolike particles at a certain transverse momentum, thus causing the reduction of the combined $v_{2}$. Therefore, we predict that elliptic flow at LHC will be smaller than that at RHIC, $v_{2}^{\mathrm{LHC}}\left(p_{T}\right)<v_{2}^{\mathrm{RHIC}}\left(p_{T}\right)$, at $p_{T} \geq 3 \mathrm{GeV} / c$.

Besides, jets also account for reversing of the mass ordering of the elliptic flow at intermediate and high transverse momenta: Here the heaviest hadrons possess the largest flow. Finally, the decays of resonances are shown to push the hadron excitation functions $v_{2} / n_{q}\left(K E_{T} / n_{q}\right)$ toward the fulfillment of the NCQ scaling. However, elliptic flow of jet particles at the LHC energy should interfere with its hydrolike counterpart already at intermediate transverse momenta. Thus, the realization of the approximate number-of-constituent-quark scaling becomes worse compared with the RHIC case.

Acknowledgments. This work was supported in part by the QUOTA Program, Norwegian Research Council (NFR) under contract No. 185664/V30, the Russian Foundation for Basic Research (Grant Nos. 08-02-91001 and 08-02-92496), Grant Nos. 107.2008.2 and 1456.2008.2 of the President of the Russian Federation, and the Dynasty Foundation. 
Influence of jets and resonance decays on the constituent quark scaling of elliptic flow 6

\section{References}

[1] Voloshin S A, Poskanzer A M, Snellings R 2008 arXiv:0809.2949 [nucl-ex]

[2] Armesto N (ed.) et al 2008 J. Phys. G: Nucl. Phys. 35054001

[3] Kestin G, Heinz U 2009 Eur. Phys. J C61 545

[4] Niemi H, Eskola K J, Ruuskanen P V 2009 Phys. Rev. C79 024903

[5] Eyyubova G et al 2009 Phys. Rev. C80 064907

[6] Lokhtin I P et al 2009 Comput. Phys. Commun. 180779

[7] Amelin N S et al 2006 Phys. Rev. C74 064901; Amelin N S et al 2008 Phys. Rev. C77 014903

[8] Lokhtin I P, Snigirev A M 2006 Eur. Phys. J C46 211

[9] PHENIX Collaboration, Adler S S et al 2003 Phys. Rev. Lett. 91182301

[10] STAR Collaboration, Adams J et al 2004 Phys. Rev. Lett. 92052302

[11] PHENIX Collaboration, Adare A et al 2007 Phys. Rev. Lett. 98162301 